\title{
Inertial confinement fusion and prospects for power production
}

\author{
C.B. Edwards ${ }^{1,2}$ and C.N. Danson ${ }^{2}$ \\ ${ }^{1}$ HiPER Project, STFC Rutherford Appleton Laboratory, UK \\ ${ }^{2}$ AWE plc, Aldermaston, UK \\ (Received 24 July 2014; revised 31 October 2014; accepted 2 December 2014)
}

\begin{abstract}
As our understanding of the environmental impact of fossil fuel based energy production increases, it is becoming clear that the world needs a new energy solution to meet the challenges of the future. A transformation is required in the energy market to meet the need for low carbon, sustainable, affordable generation matched with security of supply. In the short term, an increasing contribution from renewable sources may provide a solution in some locations. In the longer term, low carbon, sustainable solutions must be developed to meet base load energy demand, if the world is to avoid an ever increasing energy gap and the attendant political instabilities. Laser-driven inertial fusion energy (IFE) may offer such a solution.
\end{abstract}

Keywords: ICF; IFE; inertial confinement fusion; inertial fusion energy

\section{Benefits of inertial fusion energy}

Inertial fusion energy (IFE) has the potential to make a substantial contribution to meeting world energy needs in the second half of this century.

Security of supply and sustainability: IFE provides energy security and avoids geopolitical constraints because the key components of the fuel, deuterium and lithium, are abundant and widely distributed. Assessments carried out within the HiPER (European High Power Laser Energy Research Facility) project $^{[1]}$ show that sufficient materials are available for global power production at the $1 \mathrm{TWe}$ (terawatt electrical) level for more than 1000 years.

Inherent safety: IFE is intrinsically very safe since it carries no risk of 'thermal runaway'. There is little stored energy within the system, no 'critical mass' issues and, under fault conditions, energy production would simply stop.

Low environmental impact: There are no carbon emissions from the fusion energy production process. With the use of suitable materials for the reaction vessel, the relatively small amount of radioactive waste generated from neutron activation will be short lived with the appropriate choice

Correspondence to: C.N. Danson, AWE, Aldermaston, Reading, RG7 4PR, UK. Email: colin.danson@awe.co.uk of materials and managed largely through recycling. Such materials are already available.

Affordable energy: Financial modelling based on reasonable assumptions of progress during the next phase of technology development and ignition physics shows that electricity derived from laser fusion may well be cost competitive with other environmentally acceptable sources ${ }^{[2]}$, although the energy landscape in 30-50 years is uncertain and hence difficult to predict.

Separable technology: Physical separation of major systems, laser driver, reaction vessel, balance of plant, etc., allows accelerated development and reduced costs.

\section{The D-T fusion reaction}

The principle of fusion is simple, though its realization on an industrial scale suitable for commercial energy production is technologically extremely demanding. The underlying physics involves the use of powerful lasers to heat a mixture of two hydrogen isotopes, deuterium and tritium, to an extreme temperature of greater than 50 million degrees, whereupon the constituent nuclei fuse to form a helium ion (alpha particle) and a neutron, according to the reaction shown in Figure 1. In each fusion reaction, the helium ion 


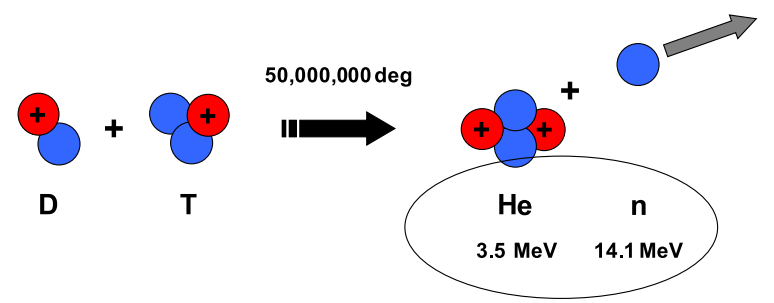

Figure 1. The D-T fusion reaction at the heart of laser energy.

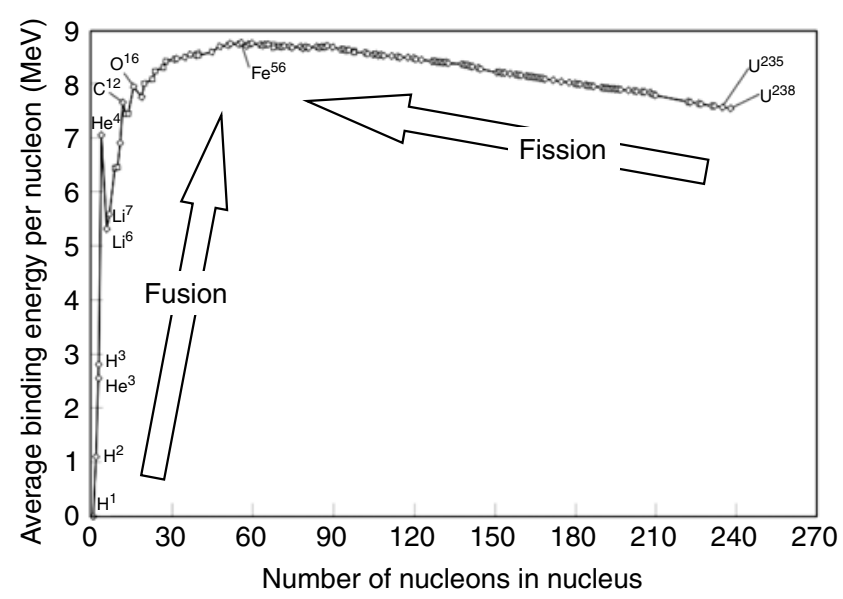

Figure 2. Binding energy variation with atomic number.

and the neutron carry excess energy totalling $17.6 \mathrm{MeV}$, millions of times greater than is liberated in a typical chemical reaction $(\sim 1 \mathrm{eV})$.

\section{The source of nuclear energy}

The energy released in nuclear fusion and fission reactions is derived from the same source. In both, the products of reaction have less mass than the constituents. The mass loss $(m)$ is released as energy $(E)$ according to Einstein's familiar equation $E=m c^{2}$, where $c$ is the velocity of light. Since the velocity of light is very large, $3 \times 10^{8} \mathrm{~m} \mathrm{~s}^{-1}$, a relatively small mass loss corresponds to a very large energy release.

This loss of mass is illustrated in graphic form in Figure 2, which plots the binding energy, or apparent loss of mass per nucleon, against atomic number. Energy release from fission corresponds to atoms of high atomic number, shown at the right-hand edge of the figure, splitting into lighter products. Fusion is represented by the joining of light elements, shown at the left-hand edge of the diagram, to produce a product of higher atomic number.

There are several potential fusion reactions, but the deuterium and tritium reaction has the highest cross section under the conditions attainable on Earth and is thus the most favourable for energy production in the foreseeable future.

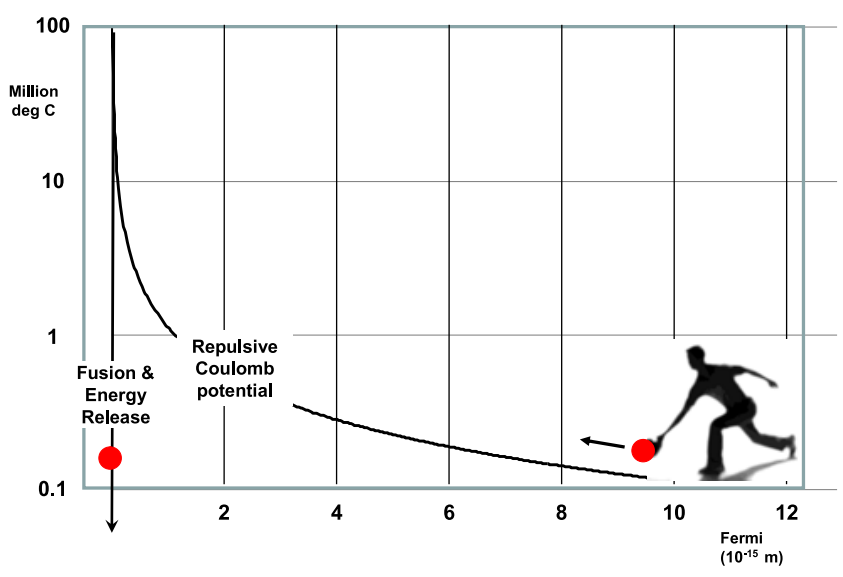

Figure 3. Potential energy schematic for fusion.

\section{Physics of thermonuclear fusion}

Although the liberated energy from both fission and fusion reactions has the same nuclear origins, there is an important difference between the physics of the two reactions which explains why power production from fusion is so technologically demanding. In the case of fission, some high atomic number nuclei are unstable and undergo spontaneous fission to produce lower atomic number products and energetic neutrons. These reactions occur at room temperature without the need to supply external energy to initiate or sustain them.

The physics of fusion is very different. When light nuclei approach to a separation comparable to their diameter, the strong nuclear force draws them even closer together until they fuse. However, this force only acts over very short distances, while at larger separation the nuclei are subjected to the repulsive Coulomb force which acts to push them apart. Only nuclei with sufficient kinetic energy to overcome the Coulomb barrier can approach closely enough to fuse. At room temperature an insignificant number of nuclei possess such energy, and external heating must be applied. It is the requirement to supply this heat energy that gives rise to the term 'thermonuclear'.

The height of the Coulomb barrier for deuterium and tritium is $1 \mathrm{MeV}$, corresponding to a temperature of 10 billion K. Fortunately, quantum mechanical tunnelling enables a significant number of neutrons to penetrate the barrier at lower energy, reducing the heating requirement to $5 \mathrm{keV}$ (50 million $\mathrm{K}$ ).

These two competing forces are shown in Figure 3, as a potential energy or 'bowling ball' diagram. The induction of fusion can be considered in terms of rolling a ball up the Coulomb potential with sufficient speed, or temperature, that it reaches the top of the barrier and falls into the potential well created by the strong nuclear force. 


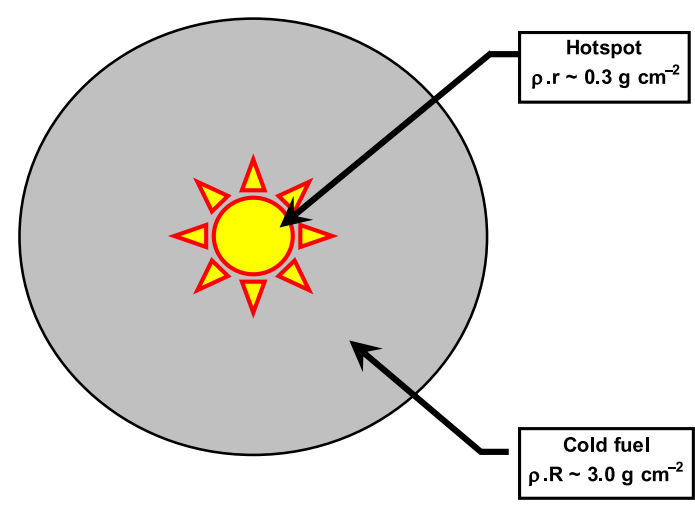

Figure 4. Assembly of the fuel at high density.

\section{Fuel assembly for a self sustaining reaction}

The fusing of nuclei by laser heating is a necessary first step in the energy production process, but this is not sufficient if the fusion process is to be efficient. The solution is to use the laser to heat a small volume of the D-T fuel, a 'hotspot', so that fusion reactions occur, and to arrange the geometry of the system so that the fusion energy released is deposited within the hotspot itself, raising the temperature further so that the fusion reactions proceed at a higher rate and heat the surrounding cold D-T fuel. This 'central hotspot ignition' approach ${ }^{[3,4]}$, shown schematically in Figure 4, can be likened to a match igniting a small amount of fuel, which then heats the surrounding material to ignition and produces a propagating burn wave.

The requirement for self heating of the hotspot region by the products of the laser-initiated reactions gives rise to a fundamental condition for efficient burning of the fuel. The $14 \mathrm{MeV}$ neutron is small, highly energetic, and typically escapes from the central hotspot of the fuel without depositing significant energy. The alpha particle is larger, less energetic and much more likely to deposit its kinetic energy within the hotspot as required. The likelihood of trapping the alpha particle depends simply on the amount of material it must penetrate before leaving the hotspot region. This can be expressed as ' $\rho \cdot r$ ' where $\rho$ is the fuel density and $r$ is the radius of the fuel in the hotspot, as shown in Figure 4. For 3.5 MeV alpha particles in a D-T mixture, the trapping condition is that $\rho \cdot r \sim 0.3 \mathrm{~g} \mathrm{~cm}^{-2}$ (Ref. [5]).

We can now investigate the energy required to heat the hotspot region to the temperature at which fusion reactions occur. Consider the hotspot in Figure 4 with radius $r$ and density $\rho$. The energy required to heat the hotspot to the temperature at which fusion reactions occur, $T_{i}$, is given by the product of the hotspot mass (volume $\times$ density) multiplied by the temperature rise $T_{i}$ and the specific heat $C_{v}$.

Thus,

$$
E=4 / 3 \cdot \pi \cdot r^{3} \cdot \rho \cdot T_{i} \cdot C_{v},
$$

which can be rearranged as

$$
E=\left[\left(4 / 3 \cdot \pi \cdot T_{i} \cdot C_{v}\right) \cdot(\rho \cdot r)^{3}\right] \cdot \rho^{-2} .
$$

However, since $\rho \cdot r$ is fixed at $0.3 \mathrm{~g} \mathrm{~cm}^{-2}$ by the alpha trapping condition, the energy can therefore be expressed as

$$
E=\text { constant } / \rho^{2} \text {. }
$$

Thus, by compressing the fuel to high density, the energy needed from the laser is reduced by the compression factor squared. For example, by compressing to a density of $\sim 500 \mathrm{~g} \mathrm{~cm}^{-3}$, compared with the normal solid density of D-T ice $\left(\sim 0.2 \mathrm{~g} \mathrm{~cm}^{-3}\right)$, the energy required to heat the fuel is reduced by a factor of $(2500)^{2}$, or six million. It is this inverse square dependence of the ignition energy with density that enables inertial fusion to be brought within the reach of currently available laser driver technology.

In principle, the laser requirement could be reduced further by compressing to even higher densities. In practice, imperfections in the uniformity of the laser beams, the ability to control the laser pulse shape and imperfections in the manufacture of the fuel capsules themselves limit the achievable density to $\sim 500 \mathrm{~g} \mathrm{~cm}^{-3}$ (Ref. [5]).

In laser driven fusion, the laser must perform two distinct functions. First, the D-T fuel must be compressed to the density required to trap the fusion alpha particles; second, the hotspot must be heated to fusion temperature, producing a burn wave that propagates through the entire fuel mass.

Great care must be taken during the compression phase, as premature heating would cause the fuel pressure to increase, requiring increased drive energy from the laser. The solution is to shape the laser pulse so that the compression occurs adiabatically, in the absence of shocks, to keep the fuel temperature low. A further issue is the control of hydrodynamic and laser-plasma instabilities (LPI) during the compression phase, also requiring increased drive. Once the fuel capsule is fully compressed, the laser power is increased rapidly to launch a number of shock waves into the fuel, which coalesce at the centre of the hotspot and raise the temperature to the ignition point. The process of compression and heating by the laser is shown schematically in Figure 5.

\section{Experimental proof of principle}

The scientific proof of principle of the basis of inertial fusion was demonstrated in a series of underground nuclear tests in the 1980s. Since that time, vigorous experimental and computational programmes have been pursued in the US, France, Japan and elsewhere to demonstrate laser-driven ignition and burn of a D-T fuel capsule.

In 2010, the 192-beam, 1.8 MJ National Ignition Facility $(\mathrm{NIF})^{[6,7]}$ was commissioned at LLNL in California with the combined mission of supporting the Stockpile 


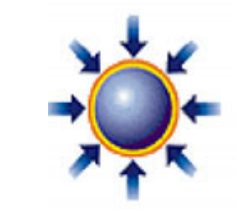

Lasers or x-rays symmetrically irradiate pellet

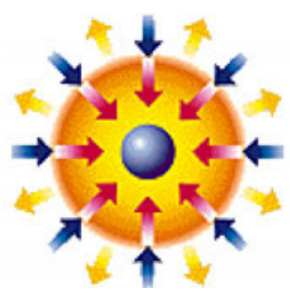

Hot plasma expands into vacuum causing shell to implode with high velocity

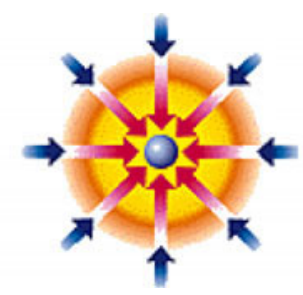

Material is compressed to $\sim 500 \mathrm{~g} \mathrm{~cm}^{-3}$

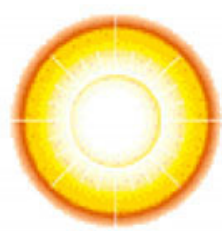

Convergence of timed shock waves ignites the core

Figure 5. Compression and heating of the fuel capsule; ignition and burn of the D-T fuel.

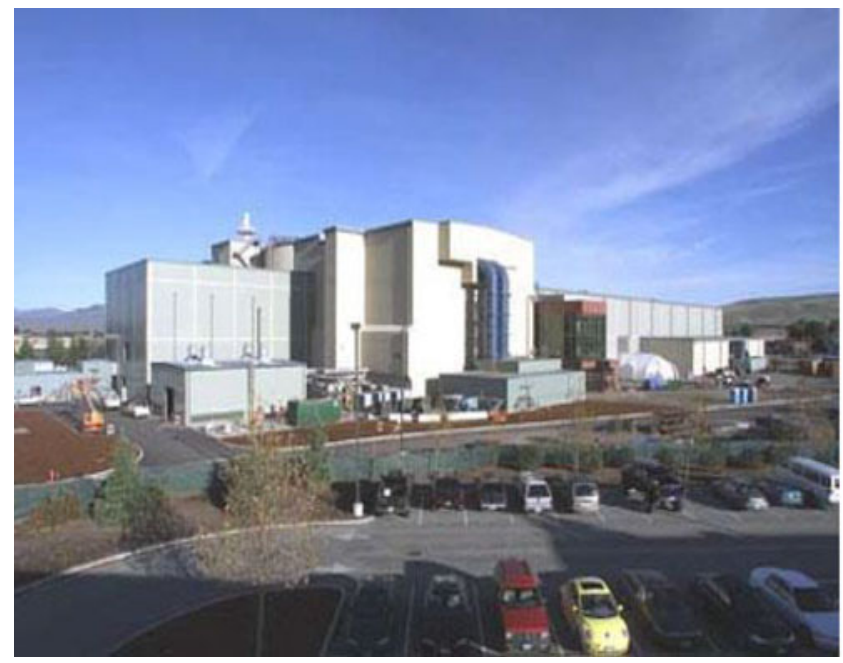

Figure 6. The NIF facility at LLNL, California, USA (picture courtesy of LLNL).

Stewardship Program, fundamental research in partnership with the academic community in the USA and elsewhere, and research into inertial confinement fusion through the National Ignition Campaign (NIC) ${ }^{[8]}$. The NIC experiments have made rapid progress towards achieving 'first ignition', and demonstration of net energy gain is anticipated within the next few years. The NIF facility building is shown in Figure 6, and Figure 7 shows one of the two NIF laser bays, each the size of a football field.

The achievement of first ignition at NIF would mark the culmination of 50 years of intensive research. It would be a full and sufficient demonstration that ICF has progressed from an elusive phenomenon of physics to a predictable, controllable technological process, ready to be harnessed for the benefit of mankind.

In 2016, the Laser Mégajoule (LMJ) facility ${ }^{[9]}$ in Bordeaux, France will be completed for start up of operations by the CEA, and ignition-scale fusion experiments can begin in Europe. A photograph of the LMJ site during the later stages of construction is shown in Figure 8. LMJ beam time will be made available to the academic community, adding additional experimental capability to the international drive for fusion.

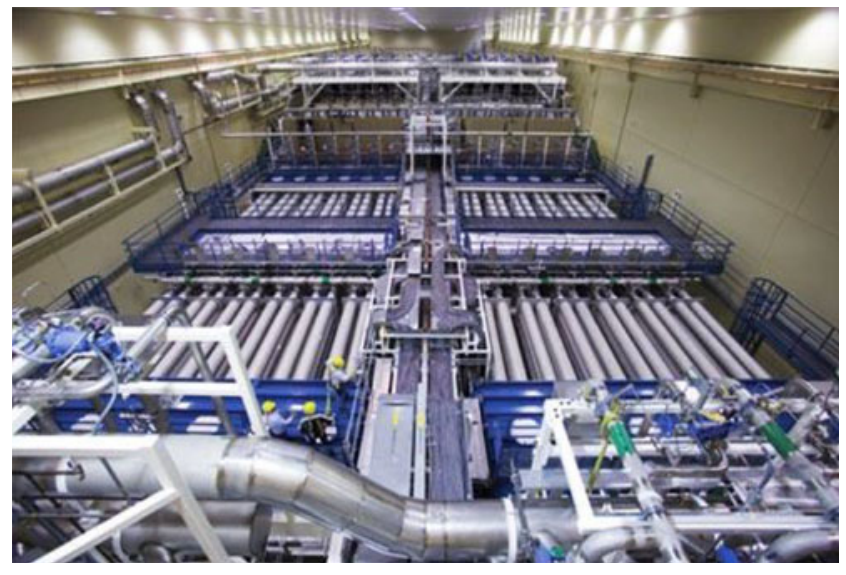

Figure 7. One of the two NIF laser bays (picture courtesy of LLNL).

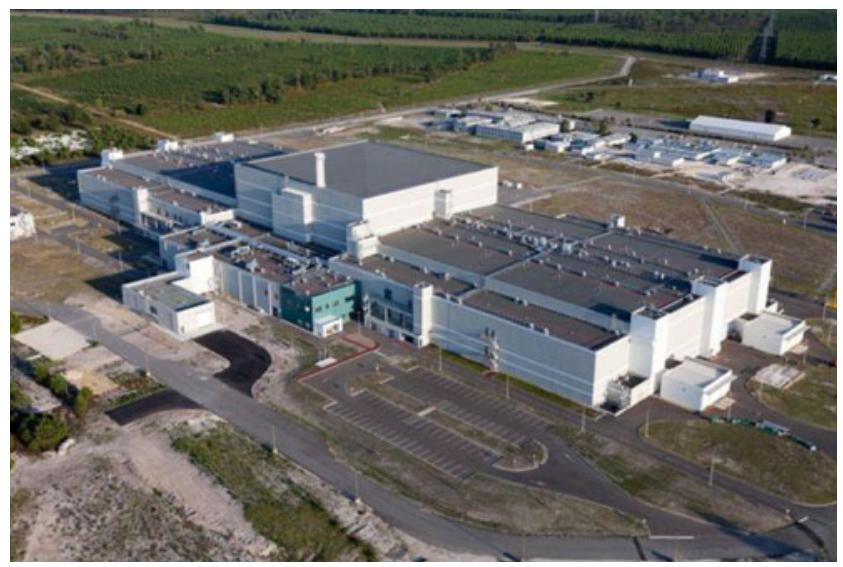

Figure 8. LMJ in Bordeaux, France (picture courtesy of CEA).

In Japan, the LFEX laser is in the final stages of commissioning and will be fully operational by 2015 . LFEX is a high energy petawatt class short pulse facility which has been built alongside the Gekko XII facility at the Institute of Laser Engineering (ILE), Osaka University ${ }^{[10,11]}$, as shown in Figure 9. The combination of high energy short pulses coupled with the long pulse Gekko XII laser facility will enable experiments to be conducted into 'fast ignition', an advanced ICF scheme which it is hoped will produce high fusion energy gain at lower laser drive energy. 


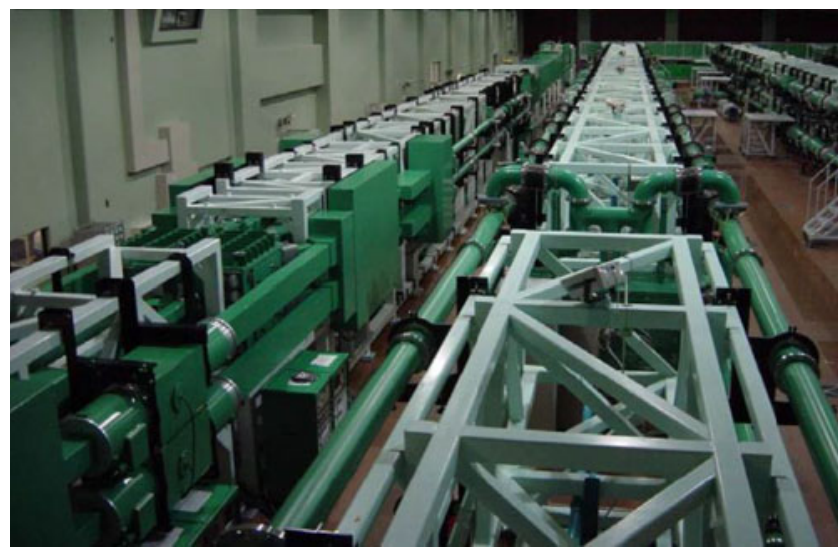

Figure 9. The Gekko XII and LFEX lasers at ILE, University of Osaka, Japan (picture courtesy of Osaka University).

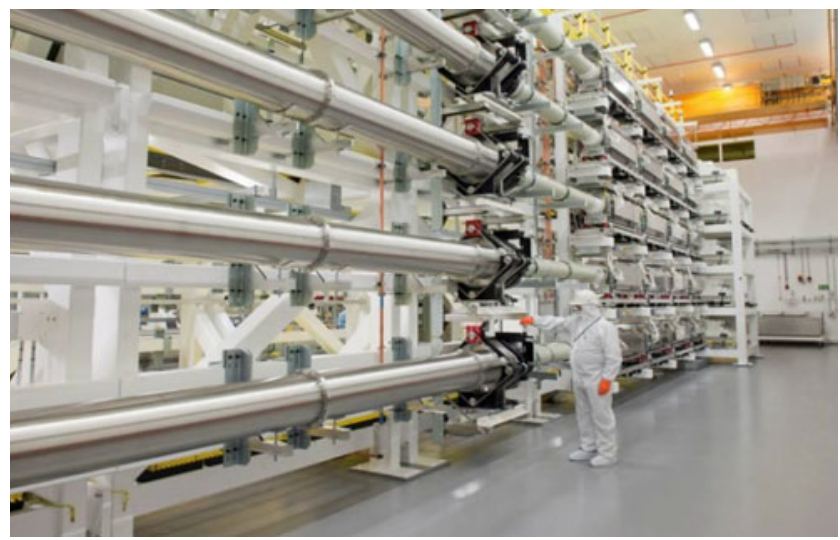

Figure 10. The laser bay of AWE's Orion facility (picture courtesy of AWE).

In the UK, scientists have access to unique, large scale, world leading laser facilities - Vulcan ${ }^{[12]}$ at Central Laser Facility (CLF), STFC Rutherford Appleton Laboratory and Orion $^{[13]}$ at AWE, as shown in Figure 10. These facilities are not powerful enough to reach the conditions for fusion gain, but they are well suited to conducting experiments designed to validate the numerical simulations on which inertial fusion depends and to investigate the underlying physics of the key processes.

UK facilities play two important roles in the laser driven fusion mission. First, the NIC in the USA has identified some areas in which the agreement between the latest experiments and simulations is poorer than expected ${ }^{[8]}$. Orion is particularly well suited to measuring the opacity and equations of state ${ }^{[14,15]}$ of materials at high density and temperature, and such measurements will help to improve the fidelity of the numerical simulations of importance to first ignition. Second, following first ignition, it will be necessary to tune the configuration of the fuel capsules and the laser parameters to optimize the energy gain of the system, to improve the robustness of ignition and to explore the sensitivity of the gain to imperfections. This will require a large number of experimental laser shots. With its high shot rate, Orion will be able to make an important contribution to this optimization process.

\section{The HiPER project}

$\operatorname{HiPER}^{[16,17]}$ is a pan-European ESFRI project (European Strategy Forum on Research Infrastructures), which seeks to demonstrate the production of secure, sustainable, safe and affordable energy with low environmental impact based on fusion driven by lasers and on a timescale relevant to meeting the challenges of the energy gap on the 2050 timescale.

Coordinated by the STFC (Science and Technology Research Council) at the Rutherford Appleton Laboratory, HiPER has created an exceptional opportunity for Europe to partner with the USA and other international partners in the approach to first ignition and to develop an international 'fast-track' approach to laser energy.

The HiPER consortium brings together 26 partners from 10 countries whose mission is to provide the scientific, technological and economic evidence to proceed to a demonstration power plant in a single step and at acceptably low commercial risk. A visualization of the HiPER concept is shown in Figure 11.

Studies of the economic viability of laser-driven fusion energy have been conducted for both HiPER and LIFE ${ }^{[18]}$, an IFE project based at LLNL. Both studies are in broad agreement, although it is acknowledged that there are difficulties in predicting economic factors 20-50 years into the future. The major conclusions are that a future plant must run at a repetition rate of at least $10 \mathrm{~Hz}$, that a fusion energy gain in excess of 60 is required and that the wall plug efficiency of the laser driver must exceed $10 \%$. The HiPER strategy is to exploit the separability of the technology by demonstrating prototypes in each of the key areas during a phase of technology development. Once this is done, construction of the HiPER power production prototype facility will present a sufficiently low technological risk to attract investment funding.

The preparatory phase of the HiPER project ended in April $2013^{[19]}$ and it has now entered its physics demonstration and technology development phase.

\section{Technical and commercial challenges ahead}

\subsection{Physics demonstration}

Demonstration of the ignition and burn of a fuel capsule, 'first ignition', at NIF, Laser Mégajoule or elsewhere is an essential precursor to major investment in the construction of a prototype power plant ${ }^{[20]}$. 


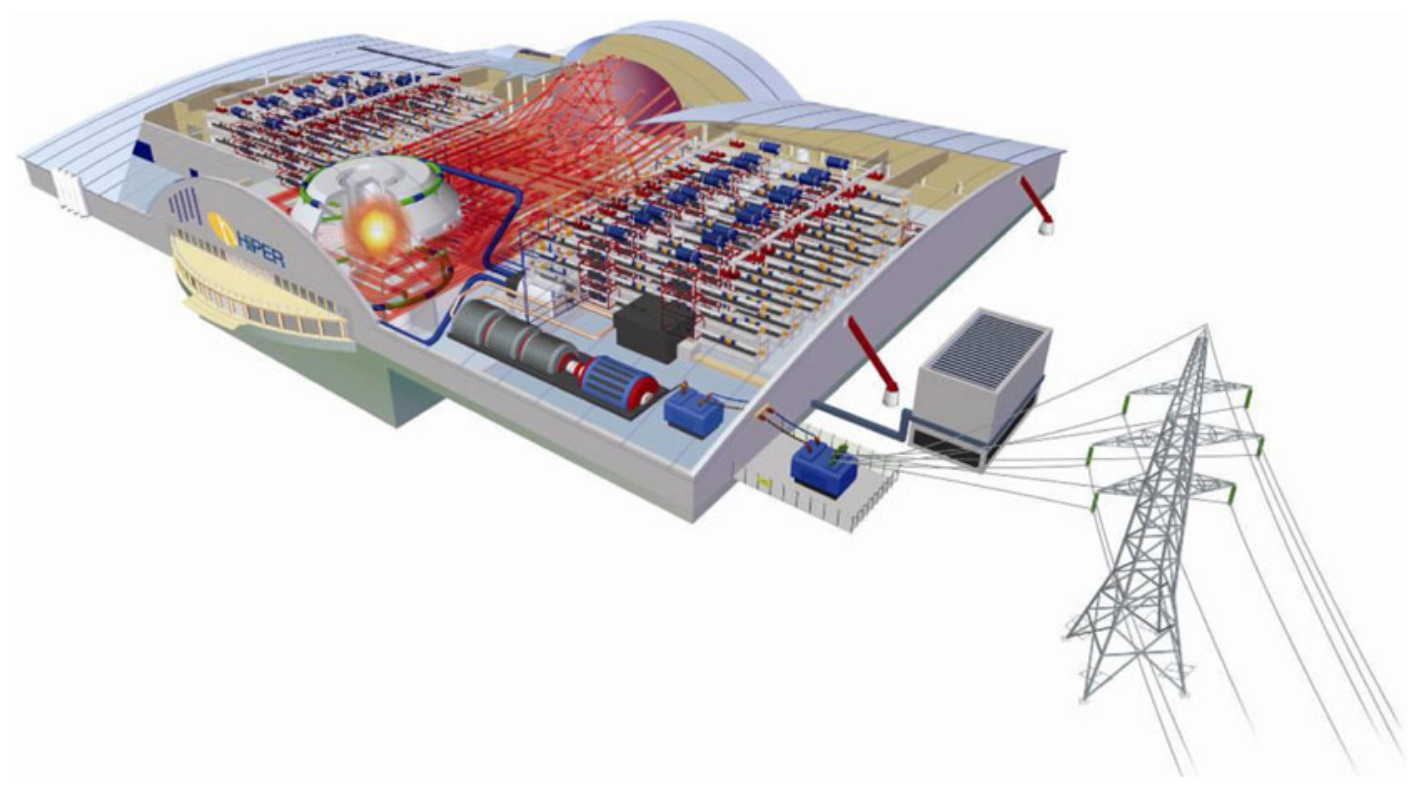

Figure 11. Visualization of the HiPER prototype facility.

The experimental programme at NIF has made exceptional progress towards first ignition in recent months, with net energy gain and indications of significant alpha particle heating of the fuel ${ }^{[21,22]}$. Further improvements are still required, particularly in the control of the hydrodynamic and LPI instabilities, but progress is encouraging and the necessary enhancements are anticipated within the next few years.

\subsection{Laser driver}

For a commercial power plant, laser driver efficiencies of $>10 \%$ and repetition rates of $>10 \mathrm{~Hz}$ will be required. The NIF laser has been designed for scientific proof of principle of laser-driven fusion on a single shot basis. With an overall efficiency of $0.1 \%$ and a repetition rate of two full energy shots per day, the technology on which NIF is based is insufficient to meet the requirement for commercial power production! A dramatic step change in laser technology is therefore clearly required to meet the driver specification. This change is now available through the use of high efficiency laser diodes to replace flashlamps as the source of the optical pump for the laser driver.

Originally developed for use in the telecommunications industry, semiconductor laser diodes provide a highly efficient means of producing very specific, 'tailored' wavelengths of light which can be used for efficient pumping of high power laser gain media. This class of device, termed 'diode pumped solid state laser' (DPSSL) ${ }^{[23]}$, offers high average power, high repetition rate operation and high efficiency. With cost reductions through mass production to meet rising demand, DPSSLs are ideal as drivers for IFE systems.
Within Europe, successful DPSSL development programmes are under way at the Central Laser Facility, STFC Rutherford Appleton Laboratory, UK ${ }^{[24]}$, at Ecole Polytechnique, Paris, France ${ }^{[25]}$, at the University of Jena, Germany ${ }^{[26]}$ and at the HiLASE Project, Prague, Czech Republic $^{[27,28]}$. All seek to demonstrate $100 \mathrm{~J} / 10 \mathrm{~Hz}$ operation at greater than $10 \%$ efficiency and all are making encouraging progress. The scaling of such systems to the $10 \mathrm{~kJ}$ level and the deployment of $\sim 200$ such beams around the fusion chamber to deliver multi-megajoule drive seems entirely feasible.

DPSSL technology has immediate exploitation potential in a wide range of industrial, medical and scientific applications. Furthermore, if the new technology can be delivered at acceptable cost, the high efficiency and low throughlife operating costs will displace existing systems while making laser treatment economically viable in new sectors. The potential economic impact of such 'next generation' laser technology in the short and medium term provides a strong driver for investment in the technology, driving up the performance of the technology while driving down the cost of installed systems.

\section{Energy capture and fuel cycle}

Deuterium is an abundant constituent of seawater and can be extracted by chemical means. Tritium, however, is radioactive with a half-life of 12.3 years and must be generated 'in situ' within the fusion fuel cycle. The process, which is based on neutron capture by lithium, is illustrated in Figure 12. Lithium is abundant and widely distributed in the Earth's crust. It can also be extracted from seawater. 


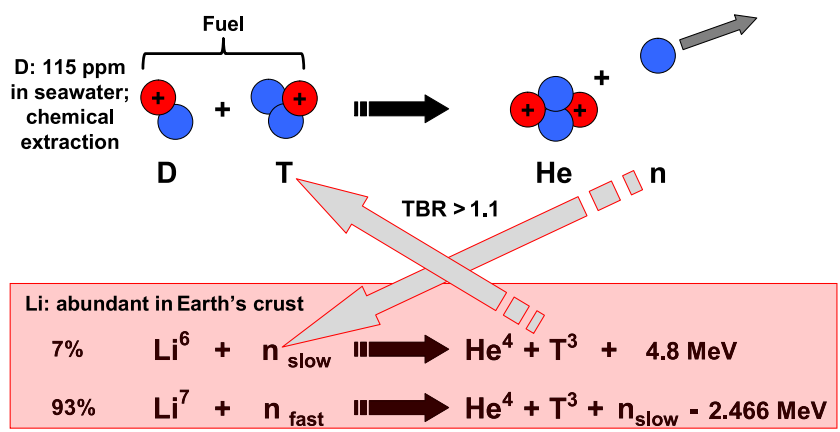

Figure 12. Neutron capture and tritium generation in lithium.

The reaction of a low energy neutron with lithium- 6 is exothermic, adding to energy production, while the lithium7 reaction is endothermic. Both reactions form tritium, and in the lithium-7 case the neutron is 'preserved' so that it can take part in a subsequent lithium- 6 reaction. Addition of lithium-7 therefore enables excess tritium to be produced to make up losses and also to generate a surplus for fuelling other fusion power plants.

The LIFE project at LLNL has the most developed concept for energy extraction, based on a simple 'boiler tube' blanket design, with a mixture of liquid lithium-6 and lithium-7 isotopes flowing in tubes around the fusion chamber and through heat exchangers.

Engineering expertise and capability associated with liquid sodium cooling of fission reactor cores are readily transferable to fusion energy plants using lithium as the primary coolant. Extraction of tritium from the liquid lithium and its recovery from the waste stream of any unburnt fuel capsules remain to be demonstrated, but, again, substantial expertise exists in the nuclear industry.

\section{Fuel capsule production}

Possibly the greatest challenge facing energy production from laser-driven fusion is the mass production of fuel capsules to the required quality and cost. Each power plant will require approximately one million fuel pellet targets per day. Commercial modelling from the HiPER preparatory phase indicates a maximum acceptable unit cost for targets of approximately 0.5 Euro/Dollars. In comparison, the current cost of the most complex NIF ignition targets, which are made individually and require many manual assembly steps, exceeds this by more than four orders of magnitude, with production times of several days. Commercial viability of laser-driven fusion power plants clearly requires a completely different approach to manufacturing techniques to increase production rates and reduce cost.

Potential process solutions have been identified, but all need development to meet the systems requirement for commercial power production. As an incentive for development, the new technologies needed for mass production of fuel capsules have very significant commercial potential. Examples include the following ${ }^{[29]}$ : advanced coating and polishing; microelectromechanical systems; microfluidics and dielectrophoresis; and the application of graphene based structures.

Microtarget design and manufacturing facilities are associated with high power laser programmes throughout the world. Together, these centres of excellence, in partnership with industry and academia, are ideally placed to make a significant contribution to the field, advancing IFE research worldwide and realizing economic impact in both the short and the long term.

\section{Conclusion}

Laser-driven IFE is based on the conversion of isotopes of hydrogen into helium through the process of fusion, using lasers as a driver. This technology could be producing energy on the 2050 timescale, with the potential to supply a significant proportion of world energy needs in the following decades.

IFE development is approaching a seminal moment, with proof-of-principle demonstration at the NIF in the USA expected within the next few years. Ignition at NIF or elsewhere will pave the way for a programme of technology development followed by construction of a prototype IFE plant to demonstrate power production on a commercially viable basis.

The HiPER project has defined a path to a commercially attractive prototype plant. The success of HiPER to date has created an exceptional opportunity for international partnerships in the run up to first ignition and thereby for the development of an international 'fast-track' approach to laser energy.

Global investment in this field over the next 5-10 years will secure an important stake in this high impact, strategic technology, providing stand-alone benefit to participating nations and positioning them as suppliers of IFE rather than customers of this highly promising technology. The net cost of such an investment would largely be offset by the value of the arising intellectual property and the economic impact of its exploitation in a diverse range of applications in the short and medium term.

\section{Acknowledgements}

The HiPER Preparatory Phase Project was supported by FP7-Infrastructures-2007-1 (Grant Agreement number 211737): 'The European High Power laser Energy Research facility Preparatory Phase Study'; the UK Science, Technology and Facilities Council; the Ministry of Education, Youth and Sports of the Czech Republic and many 'in-kind 
contributions' from the HiPER partners and their sponsoring agencies.

\section{References}

1. M. Dunne, N. Alexander, F. Amiranoff, P. Aguer, S. Atzeni, H. Azechi, V. Bagnoud, P. Balcou, J. Badziak, D. Batani, C. Bellei, D. Besnard, R. Bingham, J. Breil, M. Borghesi, S. Borneis, A. Caruso, J. C. Chanteloup, R. J. Clarke, J. L. Collier, J. R. Davies, J.-P. Dufour, P. Estraillier, R. G. Evans, M. Fajardo, R. Fedosejevs, G. Figueria, J. Fils, J. L. Feugeas, M. Galimberti, J.-C. Gauthier, A. Giulietti, L. A. Gizzi, D. Goodin, G. Gregori, S. Gus'kov, L. Hallo, C. Hernandez-Gomez, D. Hoffman, J. Honrubia, S. Jacquemot, M. Key, J. Kilkenny, R. Kingham, M. Koenig, F. Kovacs, K. Krushelnic, C. Labaune, K. Lancaster, C. Leblanc, P. H. Maire, M. Marklund, W. Martin, A. McEvoy, P. McKenna, J. T. Mendonça, J. Meyer-ter-Vehn, K. Mima, G. Mourou, S. Moustaizis, Z. Najmudin, P. Nickles, D. Neely, P. Norreys, M. Olazabal, A. Offenberger, N. Papadogianis, J.-P. Perin, J. M. Perlado, J. Ramirez, R. Ramis, Y. Rhee, X. Ribeyre, A. Robinson, K. Rohlena, S. J. Rose, M. Roth, C. Rouyer, C. Rulliere, B. Rus, W. Sandner, A. Schiavi, G. Schurtz, A. Sergeev, M. Sherlock, L. Silva, R. A. Smith, G. Sorasio, C. Strangio, H. Takabe, M. Tatarakis, V. Tikhonchuk, M. Tolley, M. Vaselli, P. Velarde, T. Winstone, K. Witte, J. Wolowski, N. Woolsey, B. Wyborn, M. Zepf, and J. Zhang, HiPER: The European High Power Laser Energy Research Facility, Technical Background and Conceptual Design Report 2007; RAL-TR-2007-008 (2007).

2. C. D. Orth, Nucl. Fusion 42, 354 (2002).

3. D. Lindl, P. Amendt, R. L. Berger, S. G. Glendinning, S. H. Glenzer, S. W. Haan, R. L. Kauffman, O. L. Landen, and L. J. Suter, Phys. Plasmas 11, 339 (2004).

4. S. H. Glenzer, B. J. MacGowan, P. Michel, N. B. Meezan, L. J. Suter, S. N. Dixit, J. L. Kline, G. A. Kyrala, D. K. Bradley, D. A. Callahan, E. L. Dewald, L. Divol, E. Dzenitis, M. J. Edwards, A. V. Hamza, C. A. Haynam, D. E. Hinkel, D. H. Kalantar, J. D. Kilkenny, O. L. Landen, J. D. Lindl, S. LePape, J. D. Moody, A. Nikroo, T. Parham, M. B. Schneider, R. P. J. Town, P. Wegner, K. Widmann, P. Whitman, B. K. F. Young, B. Van Wonterghem, L. J. Atherton, and E. I. Moses, Science 327, 1228 (2010).

5. S. Atzeni and J. Meyer-ter-Vehn, The Physics of Inertial Fusion (Oxford University Press, 2004).

6. G. H. Miller, E. I. Moses, and C. R. West, Opt. Eng. 43, 2841 (2004).

7. E. I. Moses, Nucl. Fusion 49, 104022 (2009).

8. J. Lindl, O Landen, J. Edwards, and E. Moses, Phys. Plasmas 21, 020501 (2014).

9. J. Ebrardt and J. M. Chaput, J. Phys.: Conf. Ser. 244, 032017 (2010).

10. H. Shiraga, S. Fujioka, M. Nakai, T. Watari, H. Nakamura, Y. Arikawa, H. Hosoda, T. Nagai, M. Koga, H. Kikuchi, Y. Ishii, T. Sogo, K. Shigemori, H. Nishimura, Z. Zhang, M. Tanabe, S. Ohira, Y. Fujii, T. Namimoto, Y. Sakawa, O. Maegawa, T. Ozaki, K. A. Tanaka, H. Habara, T. Iwawaki, K. Shimada, H. Nagatomo, T. Johzaki, A. Sunahara, M. Murakami, H. Sakagami, T. Taguchi, T. Norimatsu, H. Homma, Y. Fujimoto, A. Iwamoto, N. Miyanaga, J. Kawanaka, T. Jitsuno, Y. Nakata, K. Tsubakimoto, K. Sueda, N. Morio, S. Matsuo, T. Kawasaki, K. Sawai, K. Tsuji, H. Murakami, T. Kanabe, K. Kondo, R. Kodama, N. Sarukura, T. Shimizu, K. Mima, and H. Azechi, High Energy Density Phys. 8, 227 (2012).
11. H. Azechi, K. Mima, S. Shiraga, S. Fujioka, H. Nagatomo, T. Johzaki, T. Jitsuno, M. Key, R. Kodama, M. Koga, K. Kondo, J. Kawanaka, N. Miyanaga, M. Murakami, K. Nagai, M. Nakai, H. Nakamura, T. Nakamura, T. Nakazato, Y. Nakao, K. Nishihara, H. Nishimura, T. Norimatsu, P. Norreys, T. Ozaki, J. Pasley, H. Sakagami, Y. Sakawa, N. Sarukura, K. Shigemori, T. Shimizu, A. Sunahara, T. Taguchi, K. Tanaka, K. Tsubakimoto, Y. Fujimoto, H. Homma, and A. Iwamoto, Nucl. Fusion 53, 104021 (2013).

12. C. N. Danson, P. A. Brummitt, R. J. Clarke, J. L. Collier, B. Fell, A. J. Frackiewicz, S. Hancock, S. Hawkes, C. HernandezGomez, P. Holligan, M. H. R. Hutchinson, A. Kidd, W. J. Lester, I. O. Musgrave, D. Neely, D. R. Neville, P. A. Norreys, D. A. Pepler, C. J. Reason, W. Shaikh, T. B. Winstone, R. W. W. Wyatt, and B. E. Wyborn, Nucl. Fusion 44, S239 (2004).

13. N. Hopps, C. Danson, S. Duffield, D. Egan, S. Elsmere, M. Girling, E. Harvey, D. Hillier, M. Norman, S. Parker, P. Treadwell, D. Winter, and T. Bett, Appl. Opt. 52, 3597 (2013).

14. D. J. Hoarty, P. Allan, S. F. James, C. R. D. Brown, L. M. R. Hobbs, M. P. Hill, J. W. O. Harris, J. Morton, M. G. Brookes, R. Shepherd, J. Dunn, H. Chen, E. Von Marley, P. Beiersdorfer, H. K. Chung, R. W. Lee, G. Brown, and J. Emig, High Energy Density Phys. 9, 661 (2013).

15. D. J. Hoarty, P. Allan, S. F. James, C. R. D. Brown, L. M. R. Hobbs, M. P. Hill, J. W. O. Harris, J. Morton, M. G. Brookes, R. Shepherd, J. Dunn, H. Chen, E. Von Marley, P. Beiersdorfer, H. K. Chung, R. W. Lee, G. Brown, and J. Emig, Phys. Rev. Lett. 110, 265003 (2013).

16. J. Hien, L. O. Silva, G. Korn, L. A. Gizzi, and C. B. Edwards, (Eds) SPIE Volume 8080, Proceedings of 'Diode-Pumped High Energy and High Power Lasers; ELI: Ultra-relativistic Laser-Matter Interactions and Petawatt Photonics; and HiPER: the European Pathway to Laser Energy', Prague, Czech Republic, April 18, 2011.

17. http://www.hiperlaser.org.

18. M. Dunne, Fusion Sci. Technol. 60, 19 (2011).

19. http://www.hiper.org/Resources/HiPER_Preparatory_Phase_C ompletion_Report.pdf.

20. http://www.nap.edu/catalog.php?record_id=18289.

21. https://www.llnl.gov/news/newsreleases/2013/Feb/NR-13-02 $-07 . h t m l$.

22. http://www.bbc.co.uk/news/science-environment-24429621.

23. A. Tunnermann, H. Zellmer, W. Schome, A. Giesen, and K. Contag, Top. Appl. Phys. 78, 369 (2000).

24. S. Banerjee, K. Ertel, P. D. Mason, P. J. Phillips, M. Siebold, M. Loeser, C. Hernandez-Gomez, and J. L. Collier, Opt. Lett. 37, 2175 (2012).

25. J.-C. Chanteloup, A. Lucianetti, D. Albach, and T. GonçalvèsNovo, Plasma Fusion Res. 8, 3404043 (2013).

26. M. Hornung, H. Liebetrau, A. Seidel, S. Keppler, A. Kessler, J. Korner, M. Hellwing, F. Schorcht, D. Klopfel, A. K. Arunachalam, G. A. Becker, A. Savert, J. Polz, J. Hein, and C. Kaluza, High Power Laser Sci. Eng. 2, e20 (2014).

27. M. Divoky, M. Smrz, M. Chyla, P. Sikocinski, P. Severova, O. Novak, J. Huynh, S. S. Nagisetty, T. Miura, J. Pilar, O. Slezak, M. Sawicka, V. Jambunathan, J. Vanda, A. Endo, A. Lucianetti, D. Rostohar, P. D. Mason, P. J. Phillips, K. Ertel, S. Banerjee, C. Hernandez-Gomez, J. L. Collier, and T. Mocek, High Power Laser Sci. Eng. 2, e14 (2014).

28. A. Lucianetti, M. Sawicka, O. Slezak, M. Divoky, J. Pilar, V. Jambunathan, S. Bonora, R. Antipenkov, and T. Mocek, High Power Laser Sci. Eng. 2, e13 (2014).

29. M. Tolley and C. Spindloe, in Laser-Plasma Interactions and Applications, P. McKenna, D. Neely, R. Bingham and D. A. Jaroszynski, eds. Ch. 17, (Springer, 2013), p. 431, ISBN 978-3-319-00037-4. 